\title{
Sudden change of constraints in selected suspended structures
}

\author{
Wacław Szcześniak ${ }^{1}$, and Magdalena Ataman ${ }^{2, *}$ \\ ${ }^{1}$ Lublin University of Technology, Faculty of Civil Engineering and Architecture, 40 Nadbystrzycka \\ Str., 20-618 Lublin, Poland \\ ${ }^{2}$ Warsaw University of Technology, Faculty of Civil Engineering, 16 Lech Kaczyński Str., 00-637 \\ Warsaw, Poland
}

\begin{abstract}
In the paper the problem of analytic mechanics, concerning sudden change of constrains is analysed. Four rigid bodies suspended by two oblique ropes in the gravitational field are considered: the beam, the rectangular shield, the triangular shield and the circular shield. At a certain instant one rope is cut. Property of an instantaneous centre of acceleration is used to solve the problem.
\end{abstract}

\section{Introduction}

The problem of suspended rigid beam was published first by Mestchersky in [2] and by Kiedrzyński and Antoniuk in [5]. Solution of the problem was presented in [3] and [4]. Method of solution used in this items of literature resolves itself into setting of three equations of equilibrium and two geometric equations describing the centre of the beam. The equations are expressed by two angles of rotation: angle of rotation of rope and angle of rotation of beam. After derivation, and elimination of the angle of rotation of the rope, the constraint equation is obtained. This equation along with the three fundamental equations of motion allows to solve the problem.

In the paper four cases of various rigid bodies, suspended by two oblique ropes in the gravitational field, are considered. Properties of instantaneous centre of acceleration are used in the analytic solution of these problems.

\section{Problem of rigid beam}

Consider the rigid beam $\mathrm{AB}$ of mass $m$ and length $2 l$ suspended by two oblique inextensible ropes (Figure 1). At an instant rope DB is cut. The problem is to determine components of the acceleration vector of the beam midpoint $a_{x}$ and $a_{y}$, the angular acceleration $\varepsilon$ and the dynamic force $N$ in the rope FA. In this paper the method of solution will be based on the property of an instantaneous centre of acceleration. Immediately after the rope cutting the instantaneous centre of velocity is in the same point

\footnotetext{
* Corresponding author: m.ataman@il.pw.edu.p1
} 
as the instantaneous centre of acceleration. The solution is valid for $\varphi \in\left\langle 0, \frac{\pi}{2}\right\rangle$. According to the symbols in the Figure 1, we write three equations of motion, where the angle $\varphi$ is variable:

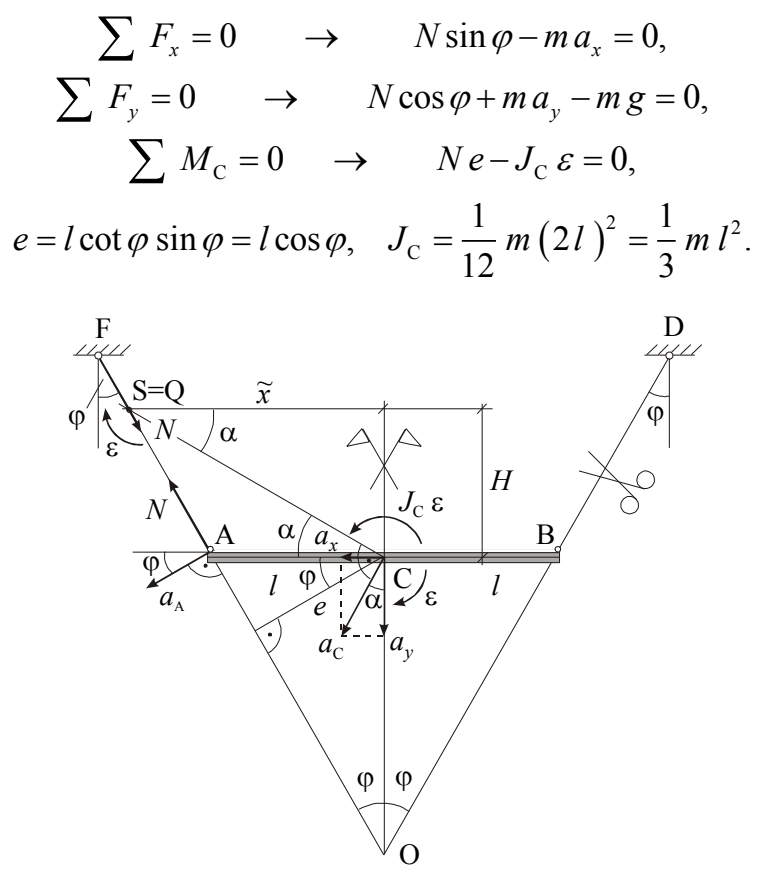

Fig. 1. Dynamic diagram of analysed rigid beam.

Equations (1) comprise four unknown quantities $a_{x}, a_{y}, \varepsilon$ and $N$. Missing equation of constraints will be determined on the basis of the Euler's lemma using property of the instantaneous centre of acceleration $\mathrm{S}=\mathrm{Q}$ the instantaneous velocity and the instantaneous angular acceleration are equal to zero at the moment. According to the symbols in the Figure 1 it can be written

$$
\begin{gathered}
\varepsilon \tilde{x}=a_{y}, \quad H=\tilde{x} \cot \varphi-l \cot \varphi, \quad H=\tilde{x} \tan \alpha=\tilde{x} \frac{a_{x}}{a_{y}}, \quad \tan \alpha=\frac{a_{x}}{a_{y}}, \\
H=H \quad \rightarrow \quad \tilde{x}=\frac{a_{y} l \cot \varphi}{a_{y} \cot \varphi-a_{x}}, \quad \varepsilon \tilde{x}=a_{y} \rightarrow \underline{l \varepsilon \cot \varphi=a_{y} \cot \varphi-a_{x}} .
\end{gathered}
$$

Solution of equations (1) and (2) is given by:

$$
\begin{array}{cc}
a_{x}=\frac{g \cos \varphi}{4 \cos \varphi \operatorname{ctg} \varphi+\sin \varphi}, & a_{y}=\frac{2 g(2+\cos 2 \varphi)}{5+3 \cos 2 \varphi}, \\
\varepsilon=\frac{6 g \cos ^{2} \varphi}{l(5+3 \cos 2 \varphi)}, & N=\frac{2 m g \cos \varphi}{5+3 \cos 2 \varphi}, \\
a_{\mathrm{C}}=\sqrt{\left(a_{x}\right)^{2}+\left(a_{y}\right)^{2}}, & a_{\mathrm{A}}=\frac{a_{x}}{\cos \varphi} .
\end{array}
$$


Above solution is illustrated in the Figure 2.

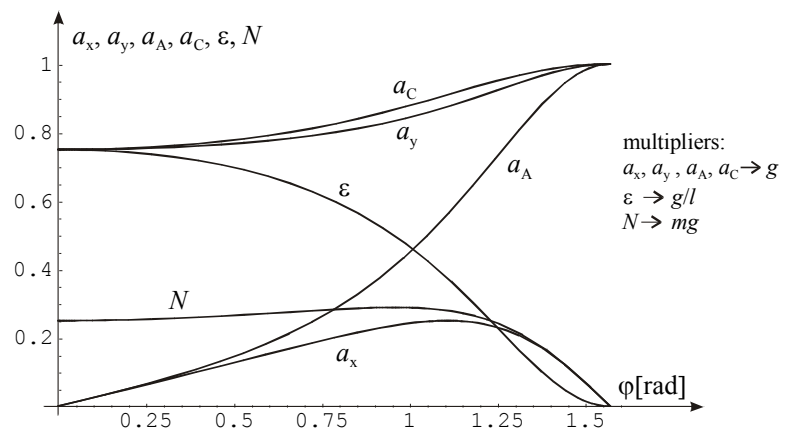

Fig. 2. Dimensionless graphs of functions $a_{x}, a_{y}, \varepsilon, N$ depending on angle $\varphi$ for rigid beam.

For $\varphi=\frac{\pi}{6}=30^{0}$ solutions (3) are reduced:

$$
a_{x}=\frac{\sqrt{3}}{13} g, \quad a_{y}=\frac{10}{13} g, \quad N=\frac{2 \sqrt{3}}{13} m g, \quad \varepsilon=\frac{9}{13} \frac{g}{l} .
$$

\section{Problem of rigid rectangular shield}

Similarly the problem of rigid rectangular shield (Figure 3) is solved. The shield is suspended by two inextensible ropes. Instantaneously the rope BD is cut. According to the symbols in the Figure 3 three standard equations of motion are written in the form:

$$
\begin{gathered}
\sum F_{x}=0 \quad \rightarrow \quad N \sin \varphi-m a_{x}=0, \\
\sum F_{y}=0 \quad \rightarrow \quad N \cos \varphi+m a_{y}-m g=0, \\
\sum M_{\mathrm{C}}=0 \quad \rightarrow \quad N e-J_{\mathrm{C}} \varepsilon=0, \\
e=\frac{1}{2}(a \cos \varphi-b \sin \varphi), \quad J_{\mathrm{C}}=\frac{m}{12}\left(a^{2}+b^{2}\right) .
\end{gathered}
$$

Equations (5) include four unknown quantities $a_{x}, a_{y}, \varepsilon$ and $N$. The fourth equation of constraints will be determined on the basis of the Euler's lemma using property of the instantaneous centre of acceleration $S=Q$ the instantaneous velocity and the instantaneous angular acceleration are equal to zero at the moment. According to the symbols in the Figure 3 it can be written

$$
\begin{gathered}
\varepsilon \tilde{x}=a_{y}, \quad H=\tilde{x} \cot \varphi-\frac{a}{2} \cot \varphi, \quad H+\frac{b}{2}=\tilde{x} \tan \alpha=\tilde{x} \frac{a_{x}}{a_{y}}, \quad \tan \alpha=\frac{a_{x}}{a_{y}}, \\
H=H \rightarrow \tilde{x}=\frac{a_{y}(b-a \cot \varphi)}{2\left(a_{x}-a_{y} \cot \varphi\right)}, \quad \varepsilon \tilde{x}=a_{y} \rightarrow \underline{\varepsilon(b-a \cot \varphi)=2\left(a_{x}-a_{y} \cot \varphi\right) .}
\end{gathered}
$$




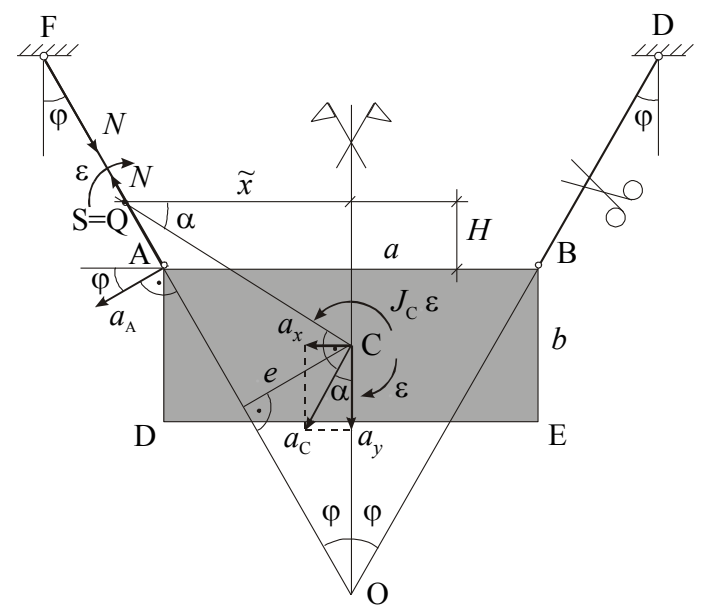

Fig. 3. Dynamic diagram of analysed rigid rectangular shield.

Solution of equations (5) and (6) is given by:

$$
\begin{aligned}
& a_{x}=\frac{2 a^{2} g \cos \varphi}{a \cos \varphi(-6 b+5 a \cot \varphi)+\left(2 a^{2}+3 b^{2}\right) \sin \varphi}, \\
& a_{y}=g-\frac{2 a^{2} g \cos \varphi \cot \varphi}{a \cos \varphi(-6 b+5 a \cot \varphi)+\left(2 a^{2}+3 b^{2}\right) \sin \varphi} \\
& \varepsilon=\frac{6 g \cos \varphi(\sin \varphi-\cos \varphi)}{a \cos \varphi(-6 b+5 a \cot \varphi)+\left(2 a^{2}+3 b^{2}\right) \sin \varphi} \\
& N=\frac{2 m g \cos \varphi}{a \cos \varphi(-6 b+5 a \cot \varphi)+\left(2 a^{2}+3 b^{2}\right) \sin \varphi}
\end{aligned}
$$

Taking into account specific case of square shield $a=b=1 \mathrm{~m}$ we obtain:

$$
\begin{aligned}
& a_{x}=\frac{2 g \cos \varphi}{-6 \cos \varphi+5 \csc \varphi}, \quad a_{y}=g+\frac{2 g \cos ^{2} \varphi}{6 \cos \varphi \sin \varphi-5}, \\
& \varepsilon=\frac{6 g \cos \varphi(\sin \varphi-\cos \varphi)}{-6 \cos \varphi+5 \csc \varphi}, \quad N=\frac{2 m g \cos \varphi}{5-6 \cos \varphi \sin \varphi} .
\end{aligned}
$$

For $\varphi=\frac{\pi}{6}=30^{0}$ the solution (7) is given by:

$$
\begin{gathered}
a_{x}=\frac{1}{73}(9+10 \sqrt{3}) g, \quad a_{y}=\frac{1}{73}(43-9 \sqrt{3}) g \\
\varepsilon=\frac{3}{73}(21-\sqrt{3}) g, \quad N=a_{y}=\frac{2}{73}(9+10 \sqrt{3}) m g
\end{gathered}
$$

In the Figure 4 kinematics and static quantities (8) depending on the angle $\varphi$ are shown. Acceleration of the shield centre $a_{C}$ is presented as well. 


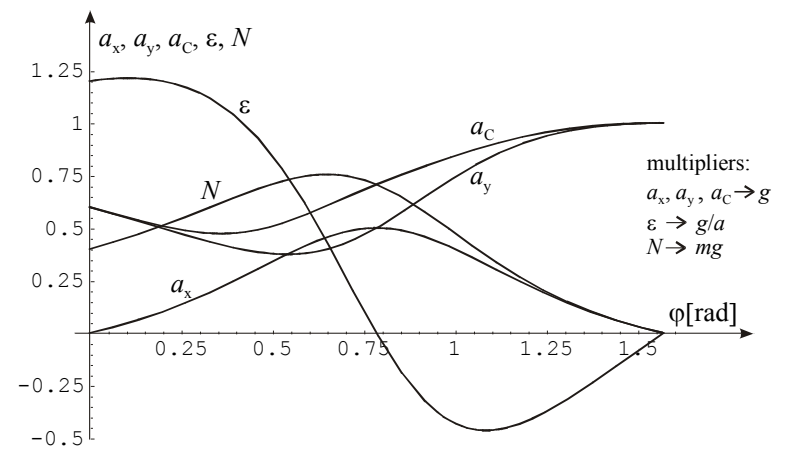

Fig. 4. Dimensionless graphs of functions $a_{x}, a_{y}, \varepsilon, N$ and $a_{C}$ depending on angle $\varphi$ for rigid square shield $a=b=1 \mathrm{~m}$.

\section{Problem of rigid triangular shield}

In case of equilateral triangle the equations of motion are given by:

$$
\begin{gathered}
\sum F_{x}=0 \quad \rightarrow \quad N \sin \varphi-m a_{x}=0, \\
\sum F_{y}=0 \quad \rightarrow \quad N \cos \varphi+m a_{y}-m g=0, \\
\sum M_{\mathrm{C}}=0 \quad \rightarrow \quad N e-J_{\mathrm{C}} \varepsilon=0, \\
e=a\left(\cos \varphi-\frac{\sqrt{3}}{3} \sin \varphi\right), \quad J_{\mathrm{C}}=\frac{m a^{2}}{3} .
\end{gathered}
$$

According to the symbols in the Figure 7 the equation of constraints is written

$$
\begin{gathered}
\varepsilon \tilde{x}=a_{y}, \quad H=\tilde{x} \cot \varphi-a \cot \varphi, \quad H=\tilde{x} \tan \alpha=\tilde{x} \frac{a_{x}}{a_{y}}, \quad \tan \alpha=\frac{a_{x}}{a_{y}}, \\
H=H \rightarrow \tilde{x}=\frac{a\left(\cot \varphi-\frac{\sqrt{3}}{3}\right) a_{y}}{a_{y} \cot \varphi-a_{x}}, \\
\varepsilon \tilde{x}=a_{y} \rightarrow \varepsilon a\left(\cot \varphi-\frac{\sqrt{3}}{3}\right)=a_{y} \cot \varphi-a_{x} .
\end{gathered}
$$

Solution the system of four equations (10) and (11) yields the expressions:

$$
\begin{gathered}
a_{x}=\frac{g \cos \varphi}{2 \cos \varphi(\sqrt{3}+2 \cot \varphi)-2 \sin \varphi}, \quad a_{y}=g+\frac{g \cos ^{2} \varphi}{-3-\cos 2 \varphi+\sqrt{3} \sin 2 \varphi}, \\
\varepsilon=\frac{g \cos \varphi(\sqrt{3} \sin \varphi-3 \cos \varphi)}{a(-3-\cos 2 \varphi+\sqrt{3} \sin 2 \varphi)}, \quad N=\frac{m g \cos \varphi}{3+\cos 2 \varphi-\sqrt{3} \sin 2 \varphi} .
\end{gathered}
$$




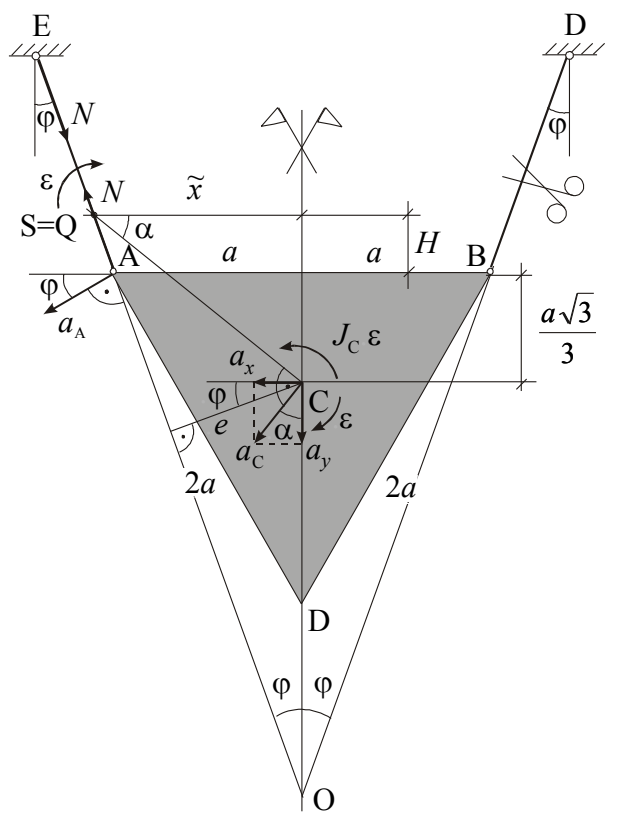

Fig. 5. Dynamic diagram of rigid triangular shield of sides $2 a$.

Assuming $\varphi=\frac{\pi}{3}=30^{\circ}$ in solution (12) yields

$$
a_{x}=\frac{3}{8} g, \quad a_{y}=\frac{5}{8} g, \quad \varepsilon=\frac{3}{4} \frac{g}{a}, \quad N=\frac{\sqrt{3}}{4} m g .
$$

Figure 6 illustrates the obtained solutions (12).

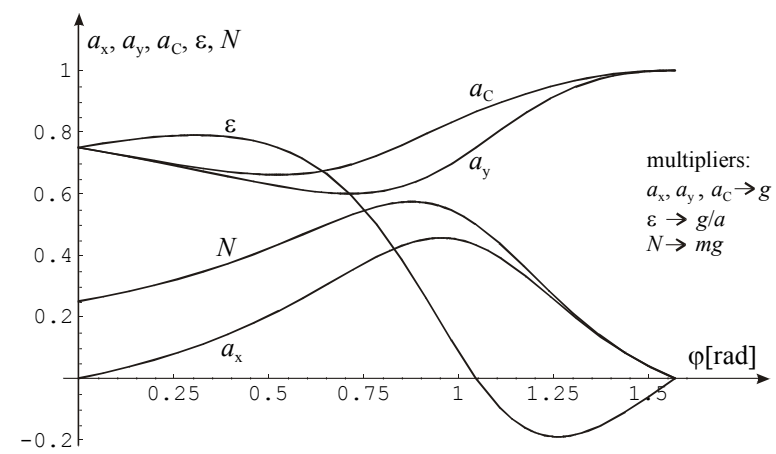

Fig. 6. Dimensionless graphs of functions $a_{x}, a_{y}, \varepsilon, N$ and $a_{C}$ depending on angle $\varphi$ for rigid triangular shield.

\section{Problem of rigid circular shield}

According to symbols in the Figure 7 the equations of motion of the circular shield are given by: 


$$
\begin{gathered}
\sum F_{x}=0 \quad \rightarrow \quad N \sin \varphi-m a_{x}=0, \\
\sum F_{y}=0 \quad \rightarrow \quad N \cos \varphi+m a_{y}-m g=0, \\
\sum M_{\mathrm{C}}=0 \quad \rightarrow \quad N e-J_{\mathrm{C}} \varepsilon=0, \\
e=R\left(\xi \cos \varphi-\sqrt{1-\xi^{2}} \sin \varphi\right), \quad J_{\mathrm{C}}=\frac{1}{2} m R^{2}=\frac{1}{3} m l^{2}, \quad A K=K B=\xi R .
\end{gathered}
$$

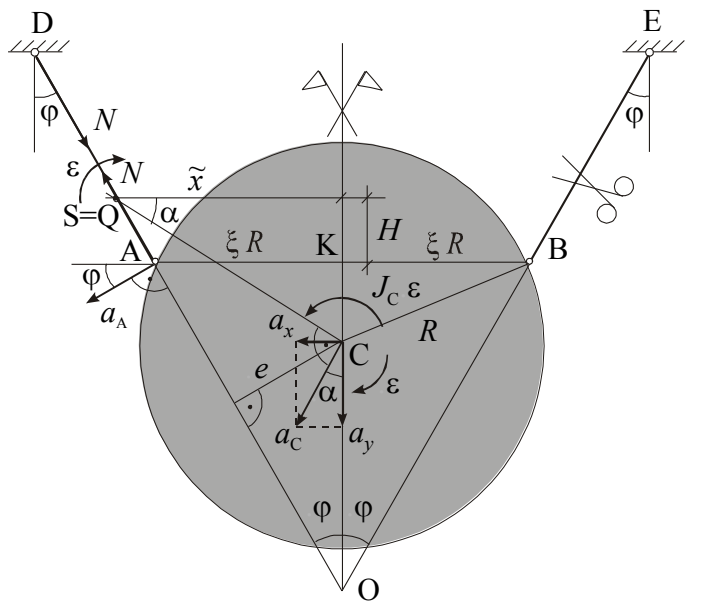

Fig. 7. Dynamic diagram of rigid circular shield.

The constraints equation for the circular shield is in the form

$$
\begin{gathered}
\varepsilon \tilde{x}=a_{y}, \quad H=\tilde{x} \cot \varphi-\xi R \cot \varphi, \quad H=\tilde{x} \operatorname{tg} \alpha=\tilde{x} \frac{a_{x}}{a_{y}}, \quad \tan \alpha=\frac{a_{x}}{a_{y}} \\
H=H \rightarrow \tilde{x}=\frac{R\left(\sqrt{1-\xi^{2}}-\xi \cot \varphi\right) a_{y}}{a_{x}-a_{y} \cot \varphi} \\
\varepsilon \tilde{x}=a_{y} \rightarrow \underline{\varepsilon R\left(\sqrt{1-\xi^{2}}-\xi \cot \varphi\right)=a_{x}-a_{y} \cot \varphi}
\end{gathered}
$$

Assuming $\xi=1 / 2$ the solution of the system of equations (14) and (15) is given by:

$$
\begin{aligned}
& a_{x}=\frac{g \sin 2 \varphi}{-4+\cos 2 \varphi+\sqrt{3} \sin 2 \varphi}, \quad a_{y}=g+\frac{2 g \cos ^{2} \varphi}{-4+\cos 2 \varphi+\sqrt{3} \sin 2 \varphi} \\
& \varepsilon=\frac{g \cos \varphi(\sqrt{3} \sin \varphi-\cos \varphi)}{R(-4+\cos 2 \varphi+\sqrt{3} \sin 2 \varphi)}, \quad N=\frac{2 m g \cos \varphi}{-4+\cos 2 \varphi+\sqrt{3} \sin 2 \varphi}
\end{aligned}
$$

In the Figure $8 a_{x}, a_{y}, \varepsilon, N$ and $a_{C}$ depending on the angle $\varphi$ are shown. 


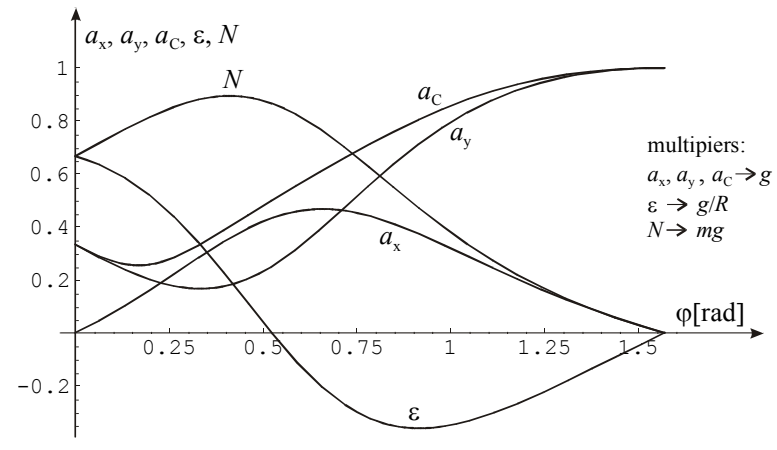

Fig. 8. Dimensionless graphs of functions $a_{x}, a_{y}, \varepsilon, N$ and $a_{C}$ depending on angle $\varphi$ for rigid circular shield.

\section{Conclusions}

In the paper four problems concerning instantaneous change of geometric constraints of structures are solved using analytic method. Properties of instantaneous centre of acceleration are used to obtain additional equation of constraints. Considered problems can be applied in analysis of a rope damage during transport of structure elements at a building site and during transport of elements in an assembly room.

\section{References}

1. W. Szcześniak, Analytic dynamics and MATHEMATICA (OWPW, Warsaw, 2005)

2. I. V. Mestchersky, Collection of problems in theoretical mechanics (Nauka, Moscow, 1986)

3. H. Neuber, Lösungen zur Aufgabensammlung Mestchersky (VEB DVW, Berlin, 1956)

4. E. H. Smart, Advanced Dynamics (Macmillan, London, 1951)

5. E. Antoniuk, A. Kiedrzyński, Problems of general mechanics. Dynamics (PWN, Warsaw, 1959)

6. R. Romicki, Solutions of problems of mechanics from the Mestchersky's collection of problems (PWN, Warsaw, 1971)

7. G. B. Karelitz, J. Ormondroyd, J. M. Garrelts, Problems in mechanics. Based on the original collection of I. V. Mestchersky (The Macmillan Co., 1939)

8. M. R. Spiegel, Theoretical Mechanics (Schaum Publishing Co., New York, 1967)

9. S. S. Rao, Mechanical Vibrations (Pearson Education, Inc., New Jersey, 2004)

10. W. Szcześniak, Theoretical dynamics for advanced (OWPW, Warsaw, 2017) 